\title{
MAGNETIC INTERACTIONS IN PRE-MAIN-SEQUENCE BINARIES
}

\author{
Fred C. Adams ${ }^{1,2}$, Michael J. Cai ${ }^{3}$, Daniele Galli ${ }^{4}$, Susana Lizano 5 , and Frank H. Shu ${ }^{3}$ \\ ${ }^{1}$ Michigan Center for Theoretical Physics Department, University of Michigan, Ann Arbor, MI 48109, USA \\ ${ }^{2}$ Astronomy Department, University of Michigan, Ann Arbor, MI 48109, USA \\ ${ }^{3}$ Institute of Astronomy and Astrophysics, Academia Sinica, Taipei 115, Taiwan, Republic of China \\ ${ }^{4}$ INAF-Osservatorio Astrofisico di Arcetri, Largo Enrico Fermi 5, I-50125 Firenze, Italy \\ ${ }^{5}$ Centro de Radioastronomía y Astrofísica, UNAM, Apartado Postal 3-72, 58089 Morelia, Michoacán, Mexico \\ Received 2011 July 25; accepted 2011 October 19; published 2011 December 2
}

\begin{abstract}
Young stars typically have strong magnetic fields, so that the magnetospheres of newly formed close binaries can interact, dissipate energy, and produce synchrotron radiation. The V773 Tau A binary system, a pair of T Tauri stars with a 51 day orbit, displays such a signature, with peak emission taking place near periastron. This paper proposes that the observed emission arises from the change in energy stored in the composite magnetic field of the system. We model the fields using the leading order (dipole) components and show that this picture is consistent with current observations. In this model, the observed radiation accounts for a fraction of the available energy of interaction between the magnetic fields from the two stars. Assuming antisymmetry, we compute the interaction energy $E_{\text {int }}$ as a function of the stellar radii, the stellar magnetic field strengths, the binary semimajor axis, and orbital eccentricity, all of which can be measured independently of the synchrotron radiation. The variability in time and energetics of the synchrotron radiation depend on the details of the annihilation of magnetic fields through reconnection events, which generate electric fields that accelerate charged particles, and how those charged particles, especially fast electrons, are removed from the interaction region. However, the major qualitative features are well described by the background changes in the global magnetic configuration driven by the orbital motion. The theory can be tested by observing a collection of pre-main-sequence binary systems.
\end{abstract}

Key words: binaries: general - magnetohydrodynamics (MHD) - stars: magnetic field - stars: pre-main sequence

\section{INTRODUCTION}

Young stars often have strong magnetic fields on their surfaces, with typical field strengths $B_{*} \sim 1-2 \mathrm{kG}$ (Johns-Krull 2009). When binary star systems have sufficiently close separation, the magnetic fields can interact over the course of the orbit. For binaries with circular orbits, variability of the radio emission should not correlate with orbital phase. For eccentric binaries, however, magnetic interactions reach a maximum near periastron and may provide a means for dissipating energy, a portion of which can be emitted as synchrotron radiation.

The T Tauri binary V773 Tau A is an observational example of such an interacting system. The stars have masses $M_{1}=1.54 \pm 0.14 M_{\odot}$ and $M_{2}=1.33 \pm 0.097 M_{\odot}$ (Boden et al. 2007). The orbit is observed to have period $P_{\text {orb }}=51.1 \pm$ 0.02 days and eccentricity $\varepsilon=0.27 \pm 0.01$. The estimated stellar radii are $R_{1}=2.22 \pm 0.20 R_{\odot}$ for the primary and $R_{2}=1.74 \pm$ $0.19 R_{\odot}$ for the secondary. This binary system is actually the "inner" binary of a more extended quadruple system; for the purposes of this paper, however, the other two components are too distant to affect the dynamics and will be ignored. With the above orbital parameters, the semimajor axis $a_{0}=0.38 \mathrm{AU}$. The separation $2 L$ between the two stars (twice the interaction distance $L$ ) equals $a_{\mathrm{o}}(1+\varepsilon) \approx 0.48 \mathrm{AU}$ at apoastron and $a_{\mathrm{o}}(1-\varepsilon) \approx 0.28 \mathrm{AU}$ at periastron. The radio flaring activity (Massi et al. 2006; Torres et al. 2011) varies from a few mJy near apoastron, where $2 L \approx 50 R_{*}$, to a few hundred mJy near periastron, where $2 L \approx 30 R_{*}$, if we adopt a mean stellar radius $R_{*}=2 R_{\odot}$.

The scenario that we envision for this source can be described as follows. Each member of the binary pair has a strong magnetic field on its surface. Young stars are often observed to have magnetic field contributions from several multipoles, with the octupole component being important near the surface
(Gregory et al. 2010); however, the dipole field dominates at the distance of the interaction region. Over the binary orbit, the distance between the stars varies in cyclical fashion. For aligned dipole fields, the magnetic fields become squeezed together as the stars become closer, and the magnetic field plays the role of a spring-like restoring force; the gravitational force is much larger and little energy is dissipated. For anti-aligned dipoles, however, the field lines can connect one star to the other, as depicted in Figure 1. The field lines that originate at high latitudes connect one star to the other, whereas the field lines that originate at low latitudes connect the star back to itself through loops that cross the equatorial plane on the sides in the opposite direction from the other star. Note that these field lines are much like that of a dipole configuration, but are distorted by the magnetic presence of the companion. When the stars are closer, more field lines that start on one stellar surface end on the other star; the total energy stored in the composite magnetic field configuration is thus smaller at periastron than for larger separations. This trend is illustrated by Figure 2, which shows 10 particular field lines when the system is at periastron (top panel) and apoastron (bottom panel). Since the stored magnetic energy changes over the orbit, it can provide power for the observed synchrotron emission. Note that energy must be given back to the magnetic field configuration as the stars move apart-work must be done on the system. Besides dynamo action in the stellar interiors, orbital motion provides the ultimate energy source for pumping up the fields after each dissipation episode. Even if the energy dissipated in heat/radiation comes entirely from the orbit, however, the implied changes in the orbital elements are too small to observe. Although this paper specializes to the case of anti-aligned dipoles, we note that reconnection is possible for more general magnetic field configurations (so that the analysis presented below is qualitatively valid). 


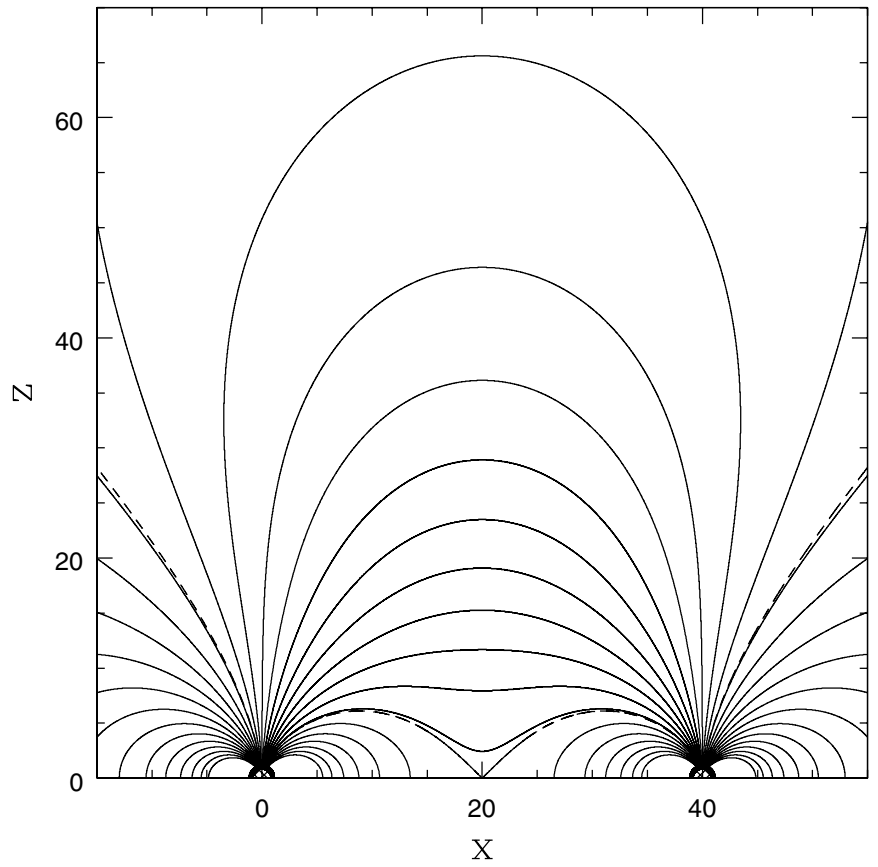

Figure 1. Magnetic field lines for an interacting binary system with separation intermediate between periastron and apoastron (in the $y=0$ plane). Lengths are given in units of $R_{*}$. The field lines are spaced at uniform intervals on the stellar surfaces. The stars have the same size $R_{*}$ and surface field strengths $B_{*}$, and have anti-aligned dipole fields. The dashed curves show the field lines that mark the boundary between those that close on the star where they originate and those that extend to the other star. These limiting field lines effectively loop to the other star via $z= \pm \infty$. (Only field lines within the plane containing the line of centers of the two stars are shown.)

Previous models of the radio emission from the V773 Tau A binary have been proposed. In particular, the emission could arise from the interaction of magnetic helmet streamers emanating from the stellar surfaces (Massi et al. 2006, 2008). Our model is similar in spirit to this previous work, except in our picture, the helmet streamers are created as a result of the interaction rather than pre-exist as independent structures that happen to "bump" when the stars pass each other at periastron. The scenario explored herein assumes that the energy comes from the large-scale field structure (dipoles), whose interaction characteristics are then computed rather than imposed.

\section{MAGNETIC INTERACTION MODEL}

\subsection{Formulation}

The two stars of V773 Tau A lack strong infrared excesses, consistent with their classification as weak-line T Tauri stars. As a first treatment, we thus assume that the space between the stars contains no dynamically significant mass from circumstellar disks or their associated winds (Königl 1991; Shu et al. 1994). Instead, we suppose that any tenuous plasma that exists above the stellar atmospheres has negligible inertia relative to the electromagnetic forces. With the magnetic fields dominant over both matter and gravity, they must satisfy the force-free condition that any currents present in the system flow parallel to the lines of force,

$$
\nabla \times \mathbf{B}=\alpha \mathbf{B},
$$

where $\alpha$ is a function of spatial position. Nonzero values of $\alpha$ correspond to non-vanishing electric current. The divergence of the above equation, and the condition of no magnetic monopoles
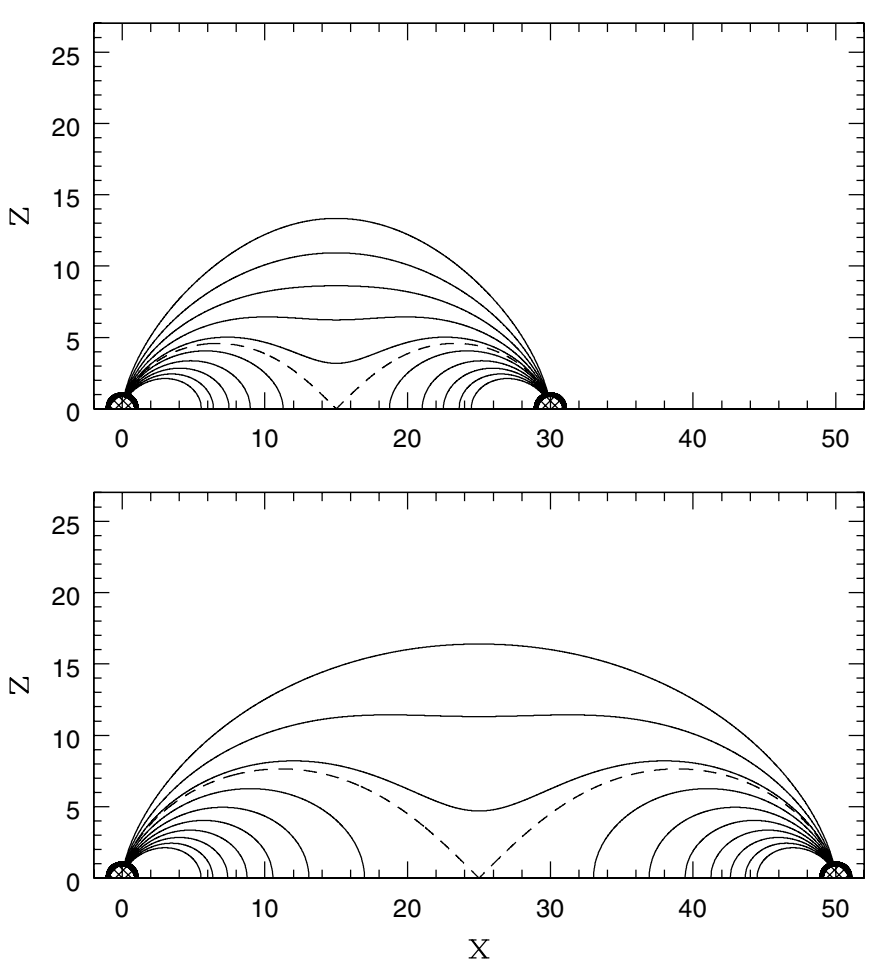

Figure 2. Magnetic field lines for an interacting binary system at periastron (top) and apoastron (bottom) in the $y=0$ plane (the plane containing the line of centers of the two stars). Ten field lines are shown (solid curves), where the fields lines start at the same polar angles on the stellar surfaces for both cases. The stars have the same size $R_{*}$ and surface field strengths $B_{*}$, and have anti-aligned dipole fields. Lengths are given in units of $R_{*}$. The figure shows that a larger fraction of the field lines connect the two stars when they are closer together (compare top and bottom panels). The dashed field line (the separatrix) starts at different angles on the two stars when the system is at apoastron and periastron. This field line has an X-point, where the field strength $\mathbf{B}$ vanishes. As the stars press together, loops below the X-point reconnect to become the fields above the X-point.

$\nabla \cdot \mathbf{B}=0$, imply that $\alpha$ satisfies the subsidiary condition B $\cdot \nabla \alpha=0$, i.e., $\alpha$ is constant along field lines.

Suppose current does not flow to/from infinity, i.e., the system neither gains nor loses net electric charge over time. Then $\alpha=0$ on open field lines and hence the open field lines must satisfy the vacuum-field equations. The closed field lines have two topological types: (1) the field lines emerge from the surface of a star and submerge beneath the surface elsewhere on the same star. (2) The field lines emanate from the surface of one star and terminate beneath the surface of the second star. In either case, in the presence of finite resistivity, any currents will dissipate with time and will asymptotically approach zero unless dynamo action restores the differential stresses on electrons and ions that led to the currents in the first place. Because current dissipation involves the transformation of field energy into heat and/or radiation (e.g., synchrotron emission if non-thermal processes of accelerating charges are involved), vacuum field configurations represent a lower state of magnetic energy than their forcefree counterparts. The important point is that current dissipation and magnetic-field reconnection tend to enforce $\alpha \rightarrow 0$, i.e., the closed field lines approach a vacuum-field configuration. For the sake of completeness we note that a pure vacuum field configuration may not be fully accessible, since finite resistivity only pushes the system toward the lowest energy state consistent with its helicity. Here, we assume that the helicity is small and consider vacuum-field configurations for both open and closed 
field lines defined by the equations

$$
\nabla \times \mathbf{B}=0 \quad \text { and } \quad \nabla \cdot \mathbf{B}=0 .
$$

We are thus computing the minimum energy states that would be present in the magnetically interacting binary system if current dissipation occurs on a rapid timescale compared to the orbital period. This philosophy informs the rest of our analysis until we get to Section 2.4.

\subsection{Energy of External Magnetic Fields}

As a starting approximation, we take all field lines to be closed, so that the magnetic field strength vanishes at infinity. In this case, the most general field configuration associated with either star is an exterior multipole expansion. A magnetic multipole of order $\ell \geqslant 1$ decreases with distance $r_{j} \equiv\left|\mathbf{r}-\mathbf{x}_{j}\right|$ from the stellar center as $r_{j}^{-(\ell+2)}$, where $\mathbf{x}_{j}$ is the stellar position $(j=1,2)$. In the V773 Tau A system, magnetic interactions occur at distances that are large compared to the stellar radii. To leading order, we can ignore all multipoles higher than the dipole component $\ell=1$. The vacuum field configuration of the two stars can then be written as

$$
\mathbf{B}=\mathbf{B}_{1}+\mathbf{B}_{2},
$$

where both stellar fields are pure dipoles with moments $\mathbf{m}_{j}=$ $m_{j} \hat{z}$,

$$
\mathbf{B}_{j}=\frac{3\left(\mathbf{m}_{j} \cdot \mathbf{r}_{j}\right) \mathbf{r}_{j}}{r_{j}^{5}}-\frac{\mathbf{m}_{j}}{r_{j}^{3}}=\frac{m_{j}}{r_{j}^{3}}\left(2 \cos \theta \hat{r}_{j}+\sin \theta \hat{\theta}_{j}\right),
$$

where $\mathbf{r}_{j}=\mathbf{r}-\mathbf{x}_{j}$. Note that this form for the dipole field and the corresponding definitions of the magnetic moments follow Jackson (1962); in particular, $m_{j}=B_{j} R_{j}^{3}$, where $B_{j}$ is the equatorial magnetic field strength on the stellar surface and $R_{j}$ is the stellar radius (for $j=1,2$ ).

Let $E_{B}$ be the energy in the magnetic field exterior to the two stars. If $V$ denotes the volume of all space excluding the two spheres instantaneously occupied by the stars, $E_{B}$ is given by

$$
E_{B}=\int_{V} \frac{B^{2}}{8 \pi} d^{3} r=\int_{V} \frac{B_{1}^{2}}{8 \pi} d^{3} r+\int_{V} \frac{B_{2}^{2}}{8 \pi} d^{3} r+\int_{V} \frac{\mathbf{B}_{1} \cdot \mathbf{B}_{2}}{4 \pi} d^{3} r .
$$

For each star, let $V_{j}$ denote the entire space excluding the volume occupied by $\operatorname{star} j$ at its instantaneous position. The total magnetic energy $E_{B}$ can be separated,

$$
E_{B}=E_{\mathrm{self}}+E_{\mathrm{int}},
$$

where $E_{\text {self }}$ represents the magnetic field energy that applies for isolated stars,

$$
E_{\mathrm{self}}=\int_{V_{1}} \frac{B_{1}^{2}}{8 \pi} d^{3} r+\int_{V_{2}} \frac{B_{2}^{2}}{8 \pi} d^{3} r
$$

and where $E_{\text {int }}$ represents the difference in magnetic field energy resulting from interaction of the stellar fields,

$$
E_{\mathrm{int}}=\int_{V} \frac{\mathbf{B}_{1} \cdot \mathbf{B}_{2}}{4 \pi}-\int_{V_{2}^{*}} \frac{B_{1}^{2}}{8 \pi} d^{3} r-\int_{V_{1}^{*}} \frac{B_{2}^{2}}{8 \pi} d^{3} r,
$$

where $V_{j}^{*}$ are the spherical volumes of the stars.
Independent of the stellar motion, $E_{\text {self }}$ is constant provided that internal magnetohydrodynamics can maintain constant magnetic dipole moments $\mathbf{m}_{j}$ within each star. This self energy $E_{\text {self }}$ provides a benchmark energy

$$
E_{\text {self }}=E_{1}+E_{2}
$$

where

$$
E_{j}=\frac{B_{j}^{2} R_{j}^{3}}{4} \int_{-1}^{1} d \mu \int_{1}^{\infty} \xi^{2} d \xi\left[1+3 \mu^{2}\right] \xi^{-6}=\frac{B_{j}^{2} R_{j}^{3}}{3},
$$

where the dimensionless variables are $\mu=\cos \theta$ and $\xi=r / R_{j}$. For the system parameters adopted below, $E_{\text {self }}=4.1 \times 10^{39} \mathrm{erg}$.

The interaction energy $E_{\text {int }}$ varies over the orbit and contributes to the observed variable radio emission, provided that the orbit is eccentric so that the separation $2 L$ changes with time. The final two terms in Equation (8) for $E_{\text {int }}$ involve the negative field energies of the individual stars in the excluded volumes of the other star; we called the sum of these two terms $E_{\text {excl }}$. The first term involves the mixed dot product, which can be positive or negative, depending on whether the magnetic fields from the two stars in the interaction region are primarily aligned $\left(\mathbf{B}_{1} \cdot \mathbf{B}_{2}>0\right)$ or anti-aligned $\left(\mathbf{B}_{1} \cdot \mathbf{B}_{2}<0\right)$; we call this term $E_{\text {mix }}$. The maximally negative interaction energy $E_{\text {int }}=E_{\text {excl }}+E_{\text {mix }}$ arises when the stellar dipoles are anti-aligned: in this case, oppositely directed field lines can squeeze together, reconnect, and release energy (ultimately to accelerate particles that create radiation). For the aligned case, the fields would dissipate at a (much) lower rate because the current density is lower.

Here we specialize to the antisymmetric case, where the stars have identical radii $R_{1}=R_{2}=R_{*}$ and magnetic dipole moments of identical strength that are parallel and antiparallel to $\hat{z}$, the unit normal of the orbital plane, i.e., $\mathbf{m}_{1}=B_{*} R_{*}^{3} \hat{z}=$ $-\mathbf{m}_{2}$. For the sake of definiteness, we adopt a standard value $B_{*}=1.5 \mathrm{kG}$ for both stars. Although Johns-Krull (2009) quotes an average magnetic field strength in T Tauri stars of $2.5 \mathrm{kG}$, this value includes all multipoles, so it still might be overly optimistic to attribute $B_{*}=1.5 \mathrm{kG}$ to the dipole component. However, both stars in the V773 Tau A system are K stars with deep outer convection zones, and have short rotation periods under 3 days (Welty 1995), properties that correlate with stronger surface field strengths. As a result, the V773 stars, which may well be pre-main-sequence progenitors of RS CnV stars, could be expected to support unusually well-ordered and coherent (although variable) fields.

We define $2 L$ to be the instantaneous center-to-center distance between the stars. The midpoint between the stars defines the origin of the coordinates. For stars of unequal masses, the origin is not at the center of mass and the frame of reference is accelerating rather than inertial. Moreover, the coordinate axes rotate at an instantaneous angular velocity $\Omega_{\mathrm{cm}}$ as the stars orbit about their center of mass. Given the rotation periods under 3 days, the spin angular velocities $\omega_{j}$ of both stars exceed $\Omega_{\mathrm{cm}}$ even at periastron. Thus, the system will probably support a unipolar inductor with a form reminiscent of the Io-Jupiter system (Goldreich \& Lynden-Bell 1969). The induced electric field could accelerate fast particles that augment the radio emission, and this process would have a periodicity correlated with the orbital phase. However, the changing magnetic field configuration, considered here, provides a larger induced electric field (see Section 2.6). As a result, we leave the unipolar inductor for future work and focus on the simpler quasi-magnetostatic concepts that we believe are responsible for the radio synchrotron emission. 
First we estimate the mixed dot-product term $E_{\text {mix }}$ as follows. For identical stars with anti-aligned magnetic dipoles, one can show that the composite magnetic field lines are symmetric with respect to the mid-plane perpendicular to the line connecting the stellar centers; this plane lies at a distance $L$ from either star. We assume that the interaction energy is given by the magnetic energy density integrated from this plane outward to infinity, i.e., the magnetic energy in this volume is lost because the fields from the two stars are connected. Introducing a form factor $f(\varpi, \varphi, z)$ to take care of the geometric vagaries of the dot product of the mixed fields, $E_{\text {mix }}$ can be written as

$$
\begin{aligned}
E_{\text {mix }} & =2 \int_{0}^{2 \pi} d \varphi \int_{L}^{\infty} d z \int_{0}^{\infty} \varpi d \varpi \frac{B_{*}^{2} R_{*}^{6}}{8 \pi} \frac{f(\varpi, \varphi, z)}{\left(\varpi^{2}+z^{2}\right)^{3}} \\
& =f_{\text {mean }}\left(\frac{B_{*}^{2} R_{*}^{6}}{24 L^{3}}\right)
\end{aligned}
$$

where we have used a cylindrical coordinate system with the $z$-axis pointing along the line connecting the stars; the meanvalue theorem allows us to take $f(\varpi, \varphi, z)$ out of the integral and replace it with an appropriate mean value $f_{\text {mean }}$.

Next we estimate the energy from the excluded volume integrals by replacing the field strength at each point in the volume by its value at the center of the sphere and then multiplying this value by the volume of the sphere. If we introduce a factor $f_{\text {excl }}$ to make the result exact, two such integrals yield

$$
E_{\mathrm{excl}}=f_{\mathrm{excl}}\left(\frac{B_{*}^{2} R_{*}^{9}}{192 L^{6}}\right) .
$$

We expect $f_{\text {excl }} \sim 1$ when $2 L \gg R_{*}$, which holds for the V773 Tau A system. However, the excluded volume energy $E_{\text {excl }}$ is smaller in magnitude than the contribution $E_{\text {mix }}$ by a factor of $\sim 8\left(L / R_{*}\right)^{3} \sim 10^{5}$ (for V773 Tau A system parameters). The excluded volume energy can thus be neglected to leading order.

Using the above estimates, the interaction energy is given numerically by

$$
\begin{aligned}
E_{\text {int }} \approx & f_{\text {mean }}\left(8.8 \times 10^{34} \mathrm{erg}\right)\left(\frac{B_{*}}{1500 \mathrm{G}}\right)^{2}\left(\frac{R_{*}}{1.4 \times 10^{11} \mathrm{~cm}}\right)^{6} \\
& \times\left(\frac{L}{2 \times 10^{12} \mathrm{~cm}}\right)^{-3} .
\end{aligned}
$$

Since $f_{\text {mean }}$ is positive for the parallel case and negative for the antiparallel case, we expect significant release of magnetic energy (yielding enhanced synchrotron radiation) only when the magnetic dipole moments of the two stars are (mostly) antialigned.

Next we determine the magnetic interaction energy by numerically evaluating the integral for the mixed dot-product term in Equation (8). Figure 3 plots the resulting dimensionless interaction energy $-E_{\text {int }} L^{3} /\left(B_{*}^{2} R_{*}^{6}\right) \approx-f_{\text {mean }} / 24$ versus the half-distance $L$ between the stars. Since this quantity has little variation with the binary separation $2 L$, the interaction energy scales as $E_{\text {int }} \propto L^{-3}$ to good approximation (consistent with the estimate from Equation (11)). Further, the inferred value of $f_{\text {mean }}$ is close to unity. As a result, the maximum energy released over the orbital period of the V773 Tau A binary is

$$
E_{\text {int }}^{\text {apo }}-E_{\text {int }}^{\text {peri }} \sim 6.1 \times 10^{34} \mathrm{erg} .
$$

Note that the energy difference of $E_{\text {int }}$ between apoastron and periastron-that available to convert into radiation-is only a

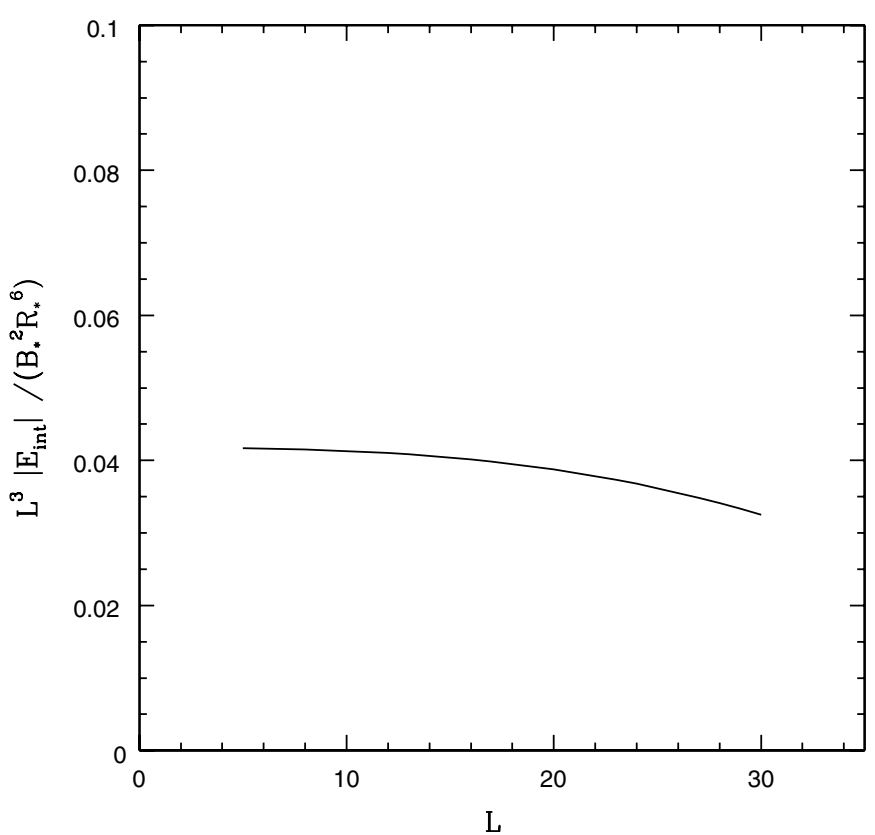

Figure 3. Dimensionless interaction energy vs. stellar half-separation, where $L$ is measured in stellar radii and the energy is scaled by $L^{3}$. The stars have the same radii and surface field strengths, and have anti-aligned dipole magnetic fields. The scaled interaction energy $L^{3} E_{\text {int }}$ varies slowly with $L$, so that $E_{\text {int }} \sim L^{-3}$.

small fraction of $E_{\text {self }}$ (about one part in $10^{5}$ ). Nevertheless, this energy difference is sufficient to explain the observed synchrotron emission.

As energy stored in the magnetic field configuration varies over the binary orbit, magnetic field lines must change their form. They become increasingly "connected" as the stars become closer (see Figure 2). The field lines that begin on one stellar surface and end on the other stellar surface generally originate near the stellar poles. The stars thus have a "polar cap" region where shared field lines begin. As the stars move farther apart (closer together), the size of the polar cap decreases (increases). Figure 4 illustrates this behavior by showing the projected polar cap for varying stellar separations. The boundaries of these polar regions are obtained by numerically integrating the magnetic field lines outward from one stellar surface to determine if the field line intersects the other stellar surface or returns to the original one. Note that the polar caps are not symmetric with respect to the geometrical pole of the star (that defined by the $\hat{z}$-axis), but rather are "tilted" toward the other star (which lies far to the right in the figure). More specifically, for each value of $y$ on the polar cap contour, there are two values of $x$. The field line that attaches to the larger algebraic value of $x$ connects to the other star through the analogue of the $\mathrm{X}$-point (drawn in Figures 1 and 2 for $y=0$ ) in the equatorial plane (also the plane of the binary orbit). The field line that attaches to the smaller algebraic value of $x$ connects to the other star through infinity (the limiting field line on the back side of the star in Figure 1 for $y=0$, i.e., the dashed curve that arches higher and higher in $z$ ). Finally, we note that Figure 4 shows that the solid angle $A_{c}$ of the polar cap is given by the approximate expression $A_{c} \approx 2 R_{*} / L$ (see Appendix A for a derivation).

\subsection{Power from Magnetic Interactions}

The above discussion estimates the magnetic interaction energy available to convert into synchrotron radiation as a function of stellar separation. The corresponding power $\mathcal{P}_{\text {mag }}$ 


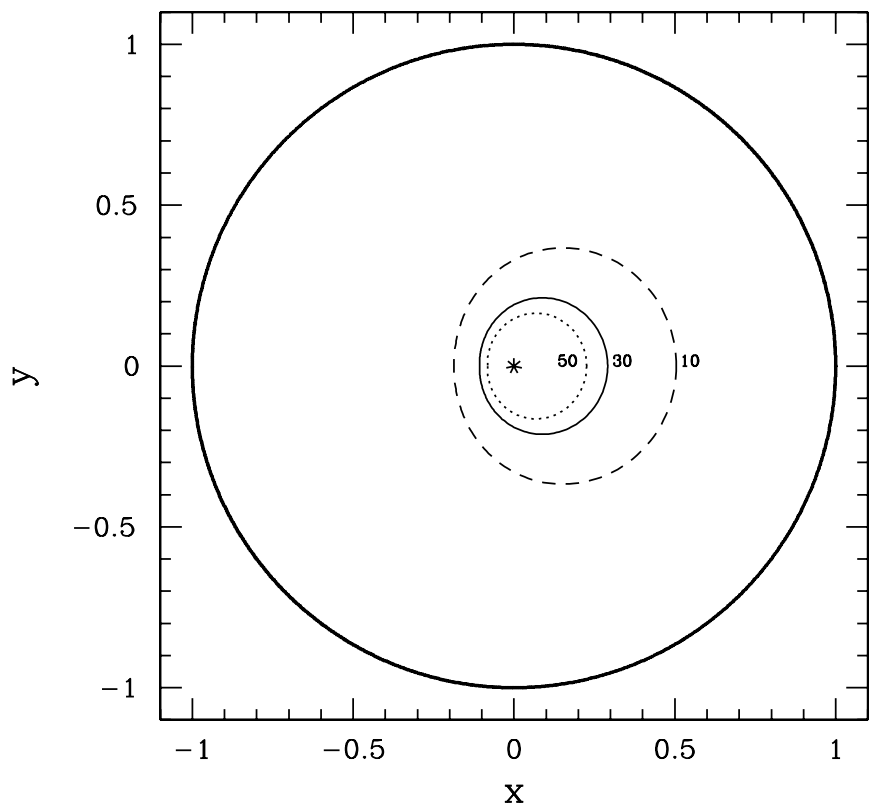

Figure 4. Polar cap region for one star of an interacting binary for varying separations. The companion star is to the right of the figure. The polar cap is the area of the star for which magnetic field lines that originate on one stellar surface terminate on the other stellar surface. The contours shown here are projections in the $x-y$ plane. There are actually two projections, one for field lines above $z=0$ (the orbital plane) and one for below $z=0$ that lie on top of each other in projection. Polar cap regions are shown for stellar separations $2 L / R_{*}=10$ (dashes), 30 (solid), and 50 (dots). The heavy circle depicts the stellar equator.

available for particle acceleration (and radiation) is the negative time rate of change of the magnetic energy and can be written as

$$
\mathcal{P}_{\text {mag }}=-\frac{d E_{\text {int }}}{d t}=3 E_{\text {int }} \frac{1}{r} \frac{d r}{d t},
$$

where $r=2 L$ is the instantaneous distance between the stars. The stars execute a Keplerian orbit,

$$
r=\frac{a_{\mathrm{o}}\left(1-\varepsilon^{2}\right)}{1+\varepsilon \cos \theta}
$$

with semimajor axis $a_{\mathrm{o}}$, eccentricity $\varepsilon$, and orbital angle $\theta$ (Murray \& Dermott 1999). Periastron occurs when $\cos \theta=$ 1. The orbital angular speed follows Kepler's second law, $\dot{\theta}=J / r^{2}$, where the specific angular momentum $J=$ $a_{\mathrm{o}}{ }^{2} \Omega\left(1-\varepsilon^{2}\right)^{1 / 2}$ and $\Omega=2 \pi / P_{\text {orb }}$ is the mean angular velocity of the orbit ( $P_{\text {orb }} \approx 51$ days). Collecting the above results, the power becomes

$$
\mathcal{P}_{\text {mag }}=f_{\text {mean }} \frac{\Omega B_{*}^{2} R_{*}^{6}}{a_{\mathrm{o}}{ }^{3}}\left(\frac{1+\varepsilon \cos \theta}{1-\varepsilon^{2}}\right)^{4} \frac{\varepsilon \sin \theta}{\left(1-\varepsilon^{2}\right)^{1 / 2}} .
$$

The positive sign in Equation (17) indicates that magnetic energy is released as the stars become closer together.

Figure 5 shows the magnitude of the total power as a function of time, which is measured in days since apoastron. The plot presents the dimensionless power $\left|\mathcal{P}_{\text {mag }} / \mathcal{P}_{0}\right|$, where we have defined the fiducial power scale

$$
\mathcal{P}_{0}=\frac{B_{*}^{2} R_{*}^{6} \Omega}{a_{\mathrm{o}}{ }^{3}} \approx 1.3 \times 10^{29} \mathrm{erg} \mathrm{s}^{-1} .
$$

Note that the power vanishes at periastron when $\sin \theta=0$, whereas peak power occurs $\sim 4$ days earlier. For simplicity, in the figure we assumed $\left|f_{\text {mean }}\right|=1$.

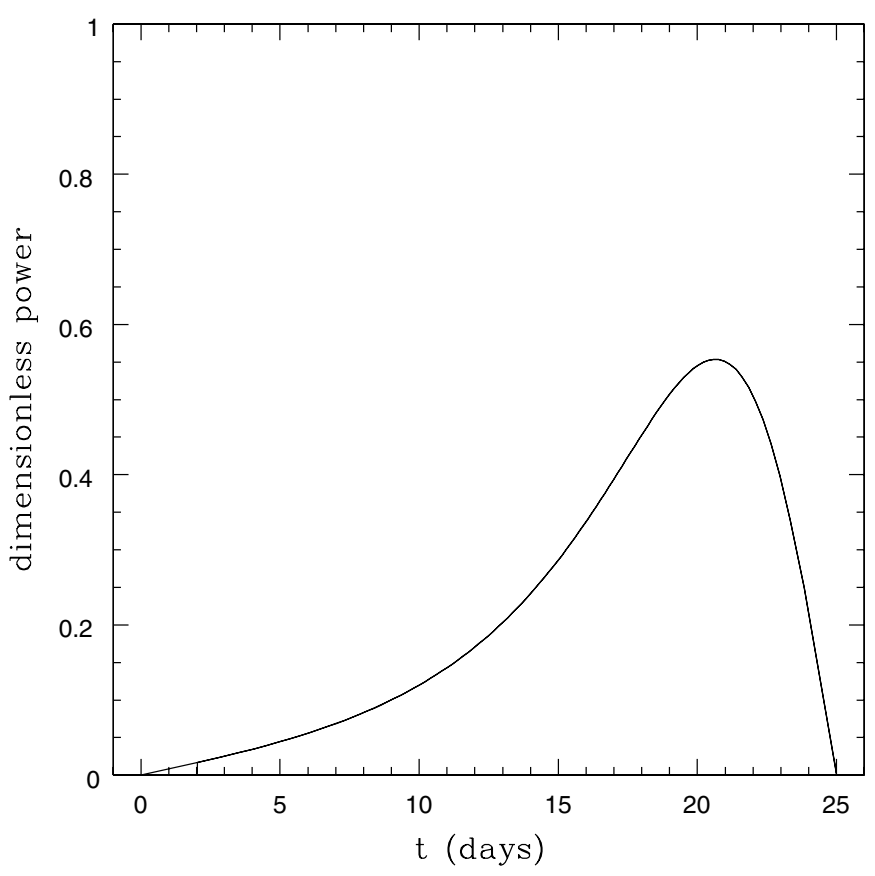

Figure 5. Power dissipated by the magnetic field configuration of the binary system over one-half orbit. Time is measured in days since apoastron. The dimensionless power $\left|\mathcal{P}_{\text {mag }} / \mathcal{P}_{0}\right|$ is plotted on the ordinate, where the fiducial power scale $\mathcal{P}_{0}$ is defined by $\mathcal{P}_{0}=B_{*}^{2} R_{*}^{6} \Omega / a_{0}{ }^{3} \approx 1.3 \times 10^{29} \mathrm{erg} \mathrm{s}^{-1}$ (see Equation (18)). Energy is dissipated over the incoming portion of the orbit.

The synchrotron emission is not necessarily fully correlated with the magnetic dissipation. Although the deformation of the magnetic field is slow and steady, with timescales characteristic of periastron passage (i.e., days; see Figure 5), the energy release from the magnetic fields and the resulting radio flares can take place on significantly shorter timescales. For example, the Sun twists up its surface fields on a natural timescale given by the differential rotation between its equator and pole (many weeks); when outbursts arise, however, they are much more rapid, lasting only minutes for impulsive flares and hours for coronal mass ejections. This phenomenon is much like the buckling of a metal plate stressed along its edges: the accumulation of stresses can be slow, but the relief of those stresses-after they become supercritical - can occur on catastrophically fast timescales.

\subsection{Acceleration of Particles to Relativistic Energies}

The loss of magnetic field energy does not directly result in radiation because the orbital frequencies, or even peak rates of reconnection, are too slow to result in meaningful electromagnetic radiation. Instead, when magnetic energies are changing because of annihilation of magnetic field $\mathbf{B}$, electric fields $\mathbf{E}$ arise that are given by Faraday's law of induction:

$$
\nabla \times \mathbf{E}=-\frac{1}{c} \frac{\partial \mathbf{B}}{\partial t}
$$

with $c$ equal to the speed of light in a vacuum. Although the resulting electric field is easy to compute as a function of space and time in the vacuum-field approximation, we do not record the results here. We merely comment that this electric field $\mathbf{E}$ does have a component parallel to the magnetic field $\mathbf{B}$, and that $\mathbf{B} \cdot \mathbf{E}$ along magnetic field lines changes sign over the orbit cycle, so that the electric currents that arise in response to the electromagnetic fields are alternating in character and do not involve a permanent charging up of either star. 
The resulting electric field $\mathbf{E}$ that is not strictly perpendicular to the magnetic field $\mathbf{B}$ then accelerates particles, in particular electrons, and it is these fast electrons, if they achieve relativistic energies, that emit synchrotron radiation in the ambient magnetic field. Some of the fast electrons may have mirror points below the surface of one of the stars in the system. In that case, electrons can spiral to the footpoints of the magnetic field and collide with ions in the dense atmosphere of the polar cap of that star. Such collisions produce brehmsstrahlung radiation, which provides another channel through which the loss of magnetic energy can appear as electromagnetic radiation.

\subsection{The Source Region for Fast Electrons}

In a fully ionized plasma, such as one that may exist in the upper chromospheric regions of the two weak-lined $\mathrm{T}$ Tauri stars, the effective frequency at which electrons are slowed down by Coulomb collisions with other charged particles decreases with increasing energy $\gamma m_{e} c^{2}$, where $m_{e}$ is the rest mass of the electron and $\gamma=\left(1-v^{2} / c^{2}\right)^{-1 / 2}$ is its Lorentz factor, and where $\mathbf{v}$ is the electron velocity. As a consequence, in the presence of a non-vanishing component $E_{\|}$of the mean electric field $\mathbf{E}$ that is parallel to the mean magnetic field $\mathbf{B}$ (i.e., $E_{\|} \equiv \mathbf{E} \cdot \hat{b}$ where $\hat{b}=\mathbf{B} /|\mathbf{B}|)$ electrons above a certain threshold energy can run away to ever higher energies (Dreicer 1959):

$$
m_{e} c^{2}\left(\frac{d \gamma}{d t}\right)_{\mathrm{elec}}=-e \mathbf{E} \cdot \mathbf{v}
$$

where $e$ is the charge of the electron and the important component of $\mathbf{v}$ in this context is that parallel to $\mathbf{B}$.

The ratio of the magnitude of the quasi-electrostatic force to the gravitational force at the poles of either star of a particle with charge $e$ and mass $m_{e}$ is $e\left|E_{\|}\right| R_{*}^{2} / G M_{*} m_{e}=\Gamma\left|E_{\|} / B_{*}\right|$ where $\Gamma \equiv e B_{*} R_{*}^{2} / G M_{*} m_{e} \sim 10^{20}$. Thus, when $\left|E_{\|}\right|$is larger than $\sim 10^{-17} B_{*}$, which is true of the vacuum field values, the electrostatic acceleration of electrons (which drag ions with them) off of the poles of one star (or the other) will more than overcome the gravitational attraction of the stars for the plasma.

Space charge can accumulate and AC current flow can occur that modify the self-consistent fields relative to the vacuum approximation of two (naked) moving magnetic dipoles. The synchrotron radiation observed in the system suggests that there are $n_{e} \sim 2 \times 10^{5}$ electrons per $\mathrm{cm}^{3}$ in a volume of space $L^{3}$ when the system is at periastron $L=15 R_{*}$. If these electrons all came from two polar caps, each of area $8 \pi R_{*}^{3} / L$ supplied at a current flow $I \sim\left(16 \pi R_{*}^{3} / L\right) e n_{e *} c$ over a time equal to half an orbital period $P_{\text {orb }} / 2$, electron number conservation requires $I P_{\mathrm{orb}} / 2=e n_{e} L^{3}$. In other words, the number density of fast electrons in the source region of the polar caps must be $n_{e *} \sim n_{e}\left(L^{4} / 8 \pi R_{*}^{3} c P_{\text {orb }}\right) \sim 426 \mathrm{~cm}^{-3}$. The magnetic field associated with the current flow $B_{\mathrm{AC}} \sim e n_{e *} R_{*} / 4 \pi \sim 2300 \mathrm{G}$ is comparable to the strength of the dipole magnetic field $2 B_{*}=3000 \mathrm{G}$ at the stellar pole. Thus, we expect the back reaction of the induced current flow of fast electrons to interfere by an order unity amount with the vacuum approximation.

An important quantity in this problem is the number density $n_{\text {fast }}$ of fast (runaway) electrons that develop when the thermal plasma of a local number density $n_{e}$ in the chromosphere or corona of one of the stars is subject to acceleration by an electric field $E_{\|}$of appropriate sign parallel to the polar-cap magnetic field of strength $2 B_{*}$. Following the treatment of Tandberg-Hanssen \& Emslie (2009), we can write the rate of fast electron production in the form

$$
\frac{d n_{\mathrm{fast}}}{d t}=0.35 n_{e} v_{c} f(\epsilon)
$$

where $\epsilon=\left|E_{\|}\right| /\left|\mathbf{E}_{D}\right|$, the strength $\left|\mathbf{E}_{D}\right|$ of the Dreicer electric field is given by

$$
\left|\mathbf{E}_{D}\right|=\frac{e}{\lambda_{D}^{2}} \ln \Lambda,
$$

and where this treatment is valid in the limit $\epsilon \ll 1$. The parameter $\lambda_{D}=\left(k T / 4 \pi n_{e} e^{2}\right)^{1 / 2}$ is the Debye length and the Coulomb $\operatorname{logarithm} \Lambda$ takes the form $\ln \Lambda=18+\ln \left(3.16 \times 10^{-5} T^{3 / 2} n_{e}^{-1 / 2}\right)$. Finally, $v_{c}$ is the electron-ion collision frequency,

$$
v_{c}=3.6 T^{-3 / 2} n_{e} \ln \Lambda,
$$

where all quantities are in cgs units. In the regime $\epsilon<1$, the function $f(\epsilon)$ has a simple form (Tandberg-Hanssen \& Emslie 2009); however, for the typical parameters of this problem $\left(n_{e} \sim 4 \times 10^{5} \mathrm{~cm}^{-3}\right.$ and $\left.T \sim 2 \times 10^{7} \mathrm{~K}\right)$, the Dreicer field $\left|\mathbf{E}_{D}\right| \sim 2 \times 10^{-11}$ statvolt $\mathrm{cm}^{-1}$, so that $\epsilon \gg 1$. In this regime almost all of the electrons are subject to runaway.

The resulting flow of current, which is almost instantaneous on the orbital timescale, will charge up both stars (one negatively and one positively), and nothing in between, because the induction electric field satisfies $\nabla \cdot \mathbf{E}=0$. The electrostatic potential associated with the negative charge grows to provide just enough repulsion of electrons (with a similar development for the positive charge on the other star in repelling ions) so that the AC current automatically shunts back and forth to maintain a quasi-steady state. In other words, a large-scale dipole electrostatic field (with one pole on each star) develops so that it compensates macroscopically for the induced electric field of the two moving magnetic dipoles. Because of the different spatial dependence of the fields of two moving electric monopoles and two moving magnetic dipoles, the cancellation of electric fields only holds as an average for the polar caps and not throughout space. Acceleration of electric charges in between the two stars occurs in response to the superposed magnetic and electric fields. In what follows, we provide order of magnitude estimates by considering only the action of the induction electric field.

\subsection{Constraints Set by the Observed Synchrotron Radiation}

To make a numerical estimate for the electron acceleration that occurs in the spaces between the two stars after the electrons leave the source and sink regions near the stellar poles, we need to specify a "typical" field strength $B_{\text {int }}$ that is undergoing change on a timescale $t_{B}$ over a length scale $L$ in the interaction region. As a typical $B_{\text {int }}$, we therefore take the average of its value at the $\mathrm{X}$-point (where $B=0$ ) and its value halfway between one of the stars and the X-point. On a direct line, this means that the distance from one star is $L / 2$ and from the other star, $3 L / 2$. For anti-aligned dipoles, the magnetic field strength at the latter point equals

$$
B_{1 / 2}=B_{*} R_{*}^{3}\left[\left(\frac{1}{L / 2}\right)^{3}-\left(\frac{1}{3 L / 2}\right)^{3}\right]=\frac{208}{27} B_{*}\left(\frac{R_{*}}{L}\right)^{3} .
$$

For our standard parameters, $B_{1 / 2} \approx 3.96 \mathrm{G}$. An average of this value and the $\mathrm{X}$-point value $\left(B_{X}=0\right)$ produces the estimate

$$
B_{\text {int }}=2 \mathrm{G} \text {. }
$$


The magnitude of the electric field is given by

$$
|\mathbf{E}| \sim\left(\frac{L}{c \tau}\right) B_{\text {int }},
$$

where $\tau$ is the timescale on which the magnetic field changes. This timescale is bounded from above by the orbit time $P_{\text {orb }} \approx$ $4.4 \times 10^{6} \mathrm{~s}$ and is bounded from below by the burst timescale $t_{B}=10^{4} \mathrm{~s}$ (from the observations of Massi et al. 2006, 2008). For all possible values of the timescale $\tau$, the length scale $c \tau \gg L=2 \times 10^{12} \mathrm{~cm}$ and hence $E \ll B_{\text {int }}$ in cgs units. Using a (conservative) value near the upper end of the range, $\tau=10^{6} \mathrm{~s}$, we find that $c \tau=3 \times 10^{16} \mathrm{~cm}$ and $|\mathbf{E}| \sim 10^{-4} B_{\text {int }}$. Nevertheless, as we shall see, the action of this electric field over the long distances available in the system can achieve impressive accelerations of charged particles.

In this work we assume that the electric field $\mathbf{E}$ generated through Faraday induction (from Equation (26)) is the dominant contribution. In particular, we verify below that $|\mathbf{E}|$ is larger than the field of a unipolar inductor, where an electric field is produced by the slippage of magnetic fields through the stellar atmosphere(s). This latter field strength, denoted here as $|\mathbf{E}|_{\text {uni }}$, can be estimated through the expression

$$
|\mathbf{E}|_{\text {uni }} \sim \frac{1}{c}|\mathbf{v} \times \mathbf{B}| \sim \frac{2 \pi R_{*}}{c P_{\text {rot }}} B_{*}\left(\frac{R_{*}}{2 L}\right)^{3},
$$

where we estimate the speed $v=|\mathbf{v}|$ of slippage as

$$
v \sim \mathcal{F} \frac{2 \pi R_{*}}{P_{\text {rot }}},
$$

where $P_{\text {rot }}$ is the rotation period of the star, and where the dimensionless fraction $\mathcal{F} \sim 1$ because the orbital angular speed of the companion star is small compared to rotation speed of the star itself. Note that to obtain this estimate, we evaluate the magnetic field strength from one star at the position of the other star. Comparing this result to the electric field obtained from Faraday induction via Equations (24)-(26), we find the ratio

$$
\frac{|\mathbf{E}|}{|\mathbf{E}|_{\text {uni }}}=\frac{416}{27 \pi \mathcal{F}} \frac{L}{R_{*}} \frac{P_{\text {rot }}}{\tau} \sim 20,
$$

where we have used $\tau=10^{6} \mathrm{~s}$ and $\mathcal{F}=1$. The electric field from Faraday induction (used here) is thus safely larger than that of the unipolar inductor, although the latter could be included as a correction in future work.

The maximum frequency of the synchrotron radiation produced by an electron with Lorentz factor $\gamma$ spiraling in a magnetic field of strength $B$ (see, e.g., Shu 1991) is given by

$$
v=\gamma^{2} v_{L}, \quad \text { where } \quad v_{L}=\frac{e B}{2 \pi m_{e} c},
$$

i.e., $v_{L}$ is the Larmor frequency. For $B=B_{\text {int }}=2 \mathrm{G}$, in order to produce $v=90 \mathrm{GHz}$ radiation, $\gamma$ must be at least 127 . Such a Lorentz factor corresponds to moderately relativistic electrons, and we may approximate $v \approx c$ when $v$ stands alone, such as in Equation (20). We now consider the length of time that a fast electron on field lines connecting the two stars can be expected to experience an electric field such that $\mathbf{E} \cdot \mathbf{v}<0$ so that it receives continuing boosts of energy via Equation (20). If the fast electron shuttles back and forth between mirror points, the maximum length of time that it spends accelerating rather than decelerating is $\sim 2 L / c$. If it is doomed to strike a polar cap before mirroring, the maximum time is again $\sim 2 L / c$. Integrating Equation (20) over the time interval $2 L / c$, we find that the maximum boost yields a Lorentz $\gamma$ given by

$$
\gamma \sim \frac{e|\mathbf{E}| 2 L / c}{m_{e} c} \sim \frac{e B_{\text {int }}(L / c \tau) 2 L}{m_{e} c^{2}} \sim 3 \times 10^{5} .
$$

In fact, such high values of $\gamma$ are inconsistent with limits obtained from considering the energy drain due to synchrotron losses. The maximum $\gamma$ consistent with synchrotron losses on a timescale $\tau=t_{B}$ is given by

$$
\gamma_{\max } \sim \frac{8 \pi m_{\mathrm{e}} c}{\sigma_{T} B_{\mathrm{int}}^{2} t_{B}} \sim 26,000,
$$

where $\sigma_{T}=8 \pi r_{\mathrm{e}}^{2} / 3=6.65 \times 10^{-25} \mathrm{~cm}^{2}$ is the Thomson cross section with $r_{\mathrm{e}}=e^{2} / m_{\mathrm{e}} c^{2}$ equal to the classical radius of the electron (see, e.g., Shu 1991). The maximum value $\gamma_{\max } \sim 26,000$ is considerably larger than the value $\gamma \sim 127$ required to produce the $90 \mathrm{GHz}$ synchrotron radiation. Having the maximum value $\gamma_{\max }$ larger than the required value of $\gamma$ is not necessarily a problem, but our arguments below suggest that the synchrotron spectrum does not extend to frequencies much higher than $90 \mathrm{GHz}$, so that some reduction of $\gamma_{\max }$ may be indicated.

Toward this end, we speculate that the self-consistent electric fields are substantially reduced by the collective response of the intervening semi-relativistic plasma, and that the energies of fast electrons have to be renewed on each periastron passage because the particles escape from the system or are lost to brehmsstrahlung as the mirror points change with reconnecting fields every orbit. The relevant decay time for the bulk of the fast electrons in the system is then $\tau \sim P_{\text {orb }}=51.1$ days $\sim 4.4 \times 10^{6} \mathrm{~s}$. The electrons that can carry over from one outburst to another have a maximum Lorentz $\gamma_{\max } \sim 59$, which implies that the electrons responsible for the $90 \mathrm{GHz}$ radiation would need re-generation for each orbit cycle. A third possibility is that the component of $\mathbf{E}$ along magnetic field lines is considerably smaller than its absolute value $|\mathbf{E}|$, so that an integral of Equation (20) produces results appreciably smaller than the naive estimate of Equation (31). We are in the process of quantitatively evaluating the cumulative result of all three effects by doing test orbit integrations and will report on the results in a future communication.

From where then does the timescale $t_{B}=10^{4} \mathrm{~s}$ arise? The timescale for magnetic reconnection is given empirically in the case of solar flares roughly by the formula:

$$
t_{B}=\frac{L}{q v_{\mathrm{A}}},
$$

where $v_{\mathrm{A}}=B_{\text {int }} /(4 \pi \rho)^{1 / 2}$ is the Alfvén speed for a plasma with mass density $\rho$ and $q$ is a dimensionless number (the Petschek parameter) whose exact value is of considerable theoretical debate but probably has a value between 0.1 and 1 . In order to achieve the burst timescale $t_{B} \sim 10^{4} \mathrm{~s}$ with $L=2 \times 10^{12} \mathrm{~cm}$, we require an Alfvén speed $v_{A}=2000 q^{-1} \mathrm{~km} \mathrm{~s}^{-1}$. With our estimate that $B_{\text {int }}=2 \mathrm{G}$, we then deduce a typical plasma density of $\rho \sim 8.0 \times 10^{-18} q^{2} \mathrm{~g} \mathrm{~cm}^{-3}$. For a fully ionized gas with solar abundance, such a plasma would have a number density of electrons given approximately by $n_{\mathrm{e}} \sim 4.2 \times 10^{6} q^{2} \mathrm{~cm}^{-3}$, well below the value, for $q \leqslant 1$, that would have caused (an unseen) Faraday de-polarization of the emitted synchrotron radiation 
(Phillips et al. 1996). Note that the communication speed $v_{\mathrm{A}}$ is large compared to the orbital speeds at which the magnetic configuration is stressed; this ordering justifies our treatment of the background magnetic evolution as being governed by elliptic partial-differential equations (the vacuum-field equations).

We still need an explanation for the value of the number density $n_{\mathrm{e}} \sim 4.2 \times 10^{6} q^{2} \mathrm{~cm}^{-3}$. The flux density $S_{v}$ at a frequency $v=90 \mathrm{GHz}$ during the radio outburst reaches a temporal peak (see Figure 2 of Massi et al. 2006) given by

$$
S_{v}=400 \mathrm{mJy}=4 \times 10^{-24} \mathrm{erg} \mathrm{s}^{-1} \mathrm{~cm}^{-2} \mathrm{~Hz}^{-1},
$$

followed by an exponential decay with mean life $2.3 \mathrm{hr}$, which we interpret to be essentially the reconnection timescale $t_{B}=10^{4} \mathrm{~s}$, and not the radio-synchrotron decay timescale $\tau \sim$ $P_{\text {orb }}=51.1$ days. We compute the total emitted synchrotron power from the product of the peak $S_{v}$ and $t_{B}=10^{4} \mathrm{~s}$ integrated over all frequencies.

To perform the integration over $v$ we have to specify the energy distribution of the relativistic electrons. In our situation, the distribution is established, not by a collisionless shock, but by the acceleration provided by magnetic reconnection that varies with the binary orbit balanced by losses through particle removal from the polar caps (and the X-region; see below). For this problem, we lack an a priori theory for the number density $n(\gamma) d \gamma$ of fast electrons with Lorentz gamma between $\gamma$ and $\gamma+d \gamma$. In Appendix B, we discuss the case of a general powerlaw distribution,

$$
n(\gamma) d \gamma=n_{0} \gamma^{-p} d \gamma
$$

For the sake of definiteness, we start by specializing to the case with $p=2.4$ (the value appropriate for collisionless shocks). For $p=2.4$, the integral of $n(\gamma) d \gamma$ from $\gamma=1$ to $\gamma=\gamma_{\max }$ is insensitive to the cutoff value $\gamma_{\max }$ as long as $\gamma_{\max } \gg 1$ (e.g., if $\left.\gamma_{\max }=127\right)$. The total number density of fast electrons in such cases is approximately $n_{0} / 1.4$.

The quantity $n_{0}$ yields the volume emissivity of synchrotron radiation as

$$
C_{1} r_{\mathrm{e}} n_{0} e B_{\perp}\left(\frac{v}{v_{\perp}}\right)^{-0.7},
$$

where $r_{\mathrm{e}}$ is again the classical radius of the electron, $v_{\perp} \equiv$ $e B_{\perp} / 2 \pi m_{\mathrm{e}} c$, and $B_{\perp}$ is the component of the magnetic field perpendicular to the line of sight (i.e., in the plane of the sky for angularly resolved observations). The dimensionless number $C_{1} \approx 4.1$ for our choice $p=2.4$, and is defined in general by Equation (B4) in Appendix B. For optically thin synchrotron emission, the flux density at a distance $d$ from the source is given by

$$
S_{v}=\left[\frac{1}{3}\left(C_{1} r_{\mathrm{e}} n_{0} e B_{\perp}\right) L\right]\left(\frac{v}{v_{\perp}}\right)^{-0.7}\left(\frac{L}{d}\right)^{2},
$$

where we have used Equation (18.20) of Shu (1991) [which is missing a factor of $R_{s}=L$ ], and where $L$ is the characteristic "radius" of the source.

With $S_{v} \propto v^{-0.7}$ in Equation (37), the implied integrated power from zero frequency to $v$ is given by

$$
\mathcal{P}_{\nu}=4 \pi d^{2} \int_{0}^{v} S_{\nu} d v=4 \pi d^{2}\left(\nu S_{v} / 0.3\right),
$$

where $d=133 \mathrm{pc}$ is the distance to V773 Tau (Torres et al. 2011). If we put in the observed value $S_{v}=400 \mathrm{mJy}=$
$4 \times 10^{-24} \mathrm{erg} \mathrm{cm}^{-2} \mathrm{~s}^{-1} \mathrm{~Hz}^{-1}$ at $v=90 \mathrm{GHz}$, we find that $\mathcal{P}_{\nu}=2.5 \times 10^{30} \mathrm{erg} \mathrm{s}^{-1}$ for the synchrotron emission from zero frequency to $v=90 \mathrm{GHz}$. Over a burst time of $t_{B}=10^{4} \mathrm{~s}$, the released energy at radio frequencies up to $90 \mathrm{GHz}$ equals $2.5 \times 10^{34} \mathrm{erg}$, which is nominally $40 \%$ of the total magnetic energy available in our model in going from apoastron to periastron, $E_{\text {int }}^{\text {apo }}-E_{\text {int }}^{\text {peri }}=6.1 \times 10^{34} \mathrm{erg}($ refer to Equation (14)).

The above considerations suggest that if $B_{*}=1500 \mathrm{G}$ is a good estimate for the stellar surface fields, the conversion of magnetic energy into synchrotron radiation must be efficient, and little synchrotron radiation is emitted at frequencies higher than $90 \mathrm{GHz}$. Because the collisional losses to the thermal plasma are expected to be negligible (see the discussion in Appendix A of Massi et al. 2006), we believe the acceleration of runaway electrons to be highly efficient, not only in the sense that a high proportion of eligible plasma can be driven to relativistic energies, but also to the extent that there is a limited escape of such accelerated electrons from the system before they are overtaken by synchrotron losses. In other words, as long as the cosmic rays generated within closed field lines do not open such field lines, almost the entire energy available from the change of the magnetic configuration will be used to accelerate the particles responsible for the deduced radio emission. Moreover, these particles essentially lose all their energy by synchrotron emission before the next cycle of field dissipation, particle acceleration, and synchrotron emission; as a result, almost all the energy input from magnetic field dissipation in the V773 Tau A system is converted into radio synchrotron emission. There is, however, a supra-thermal population of electrons that carries over from one cycle to another that serves as the seed population (the "storage ring") for the next round of synchrotron acceleration. In fact, we argue below that the supra-thermal population may be the predominant fraction of the plasma density that we estimated using the reconnection time argument.

For $B_{\perp}=2 \mathrm{G}$ and $v / v_{\perp}=(127)^{2}$, we compute from Equation (37) and $S_{v}=400 \mathrm{mJy}$ at $v=90 \mathrm{GHz}$ that $n_{0} / 1.4=1.44 \times 10^{5} \mathrm{~cm}^{3}$. Within the uncertainties of the calculation, the number density $n_{\text {fast }}$ of fast electrons needed to account for the observed radio emission is then comparable to the total electron density $n_{\mathrm{e}}=4.2 \times 10^{6} q^{2} \mathrm{~cm}^{-3}$ deduced to be present on the basis of the reconnection time. (The latter calculation was performed non-relativistically, which is justified for the ions that provide the bulk of the mass density. The number density of the electrons, which may be relativistic, is then deduced on the basis of charge neutrality.) In other words, the supra-thermal population may be a significant fraction or even all of the plasma electrons (if $q=0.19$ ), which provides the sought-for explanation for the value of $n_{\mathrm{e}}$. As a bonus, we obtain the attractive suggestion that the supra-thermal electrons may themselves be the contributor to the "anomalous resistivity" that triggers the reconnection event.

That such a source of anomalous resistivity is needed can be seen by computing the reconnection scale if it occurs by collisional resistivity: $\ell_{R}=\left(t_{B} \eta\right)^{1 / 2} \sim 3 \mathrm{~km}\left(T / 10^{4} \mathrm{~K}\right)^{-3 / 4}$, where we have used the Spitzer (1978) formula for the resistivity of a fully ionized cosmic plasma: $\eta=10^{13} T^{-3 / 2} \mathrm{~cm}^{2} \mathrm{~s}^{-1}$. For any plausible value of the temperature $T$ of a thermal plasma, the length scale $\ell_{R}$ over which collisional resistivity can induce reconnection is much smaller than the scale $L$ over which the spatially resolved very long baseline interferometry (VLBI) observations require the events to occur (Massi et al. 2006). We conclude that the true resistivity occurs not by physical 
collisions knocking charged particles off field lines, but by their scattering off fluctuating electromagnetic fields that arise because the laminar collisionless flow (particularly the counterstreaming of charges driven by quasi-electrostatic fields from the polar caps of the two stars) is unstable to the generation of plasma waves.

We can now provide a consistency check on our deduced value of $n_{0}$ using the following mechanical argument. If we follow convention and assume that the momentum distribution of cosmic-ray electrons is isotropic, we can define a cosmic-ray electron pressure $P_{\mathrm{cr}}$ through the expression

$$
\begin{aligned}
P_{\text {cr }} & =\frac{1}{3} \int_{0}^{\infty} v p n(\gamma) d \gamma \approx \frac{1}{3} m_{\mathrm{e}} c^{2} n_{0} \int_{0}^{\infty} \gamma^{-1.4} d \gamma \\
& \approx 0.83 m_{\mathrm{e}} c^{2} n_{0},
\end{aligned}
$$

where we have extended the integration to infinity because most of the contribution comes from low energies, and where we have made the approximation that the product of speed and momentum, $v p$, for a relativistic electron equals its energy $\gamma m_{\mathrm{e}} c^{2}$. Using $n_{0} / 1.4=1.44 \times 10^{5} \mathrm{~cm}^{-3}$, we find that the electron pressure $P_{\text {cr }} \approx 0.14 \mathrm{erg} \mathrm{cm}^{-3}$. This value should be compared with the magnetic pressure $B_{\text {int }}^{2} / 8 \pi \approx 0.16 \mathrm{erg} \mathrm{cm}^{-3}$. The pressure of fast electrons at peak outburst is thus comparable to the typical magnetic field pressure in the interaction region, implying that the electrons that escape from beneath the $\mathrm{X}$-point into the region above the X-point in Figure 2 can inflate the fields in the latter until they become open. Once every orbit cycle, the fast electrons can escape their confinement through such a mechanism; as a result, the density $n_{0}$ cannot accumulate through repeated accelerations to a value that is much in excess of that calculated here. Similar considerations in other contexts may offer an explanation for why the assumption of rough equipartition of energies in cosmic-ray electrons and magnetic fields holds in many systems of interest in astrophysics.

For sufficiently low frequencies, synchrotron emission is suppressed due to the Razin-Tsytovich effect (e.g., Razin 1960; Tsytovich 1951; Simon 1969; Melrose 1972). The lowest possible value of the frequency for which waves can propagate is given by

$$
v_{R}=\frac{4 \pi v_{P}^{2}}{3 \omega_{L} \sin \theta} \sim 20 \frac{n_{e}\left(\mathrm{~cm}^{-3}\right)}{B(\mathrm{G})} \mathrm{Hz} \sim 42 q^{2} \mathrm{MHz},
$$

where $v_{P}=\left(n_{e} e^{2} / \pi m_{e}\right)^{1 / 2}, \omega_{L}=e B / m_{e} c$, and where we have used $n_{e}=4.2 \times 10^{6} q^{2} \mathrm{~cm}^{-3}, B \sim 2 \mathrm{G}$, and $\sin \theta \sim 1$. This frequency is much lower than the $90 \mathrm{GHz}$ synchrotron emission observed in the V773 binary system, so that Razin-Tsytovich suppression does not appear.

We check now whether the modeled synchrotron emission is optically thin as assumed. The mean synchrotron optical depth $\tau_{v}$ through the source region is given by

$$
\tau_{v}=\frac{1}{3} C_{2} n_{0}\left(\frac{c r_{\mathrm{e}}}{v_{\perp}}\right) L\left(\frac{v}{v_{\perp}}\right)^{-3.2},
$$

where the constant $C_{2} \approx 9.9$ for $p=2.4$, and is defined in general by Equation (B9) in Appendix B. The right-hand side of Equation (41) nominally equals $5 \times 10^{-5}$ at $90 \mathrm{GHz}$, which would indeed make the synchrotron radiation optically thin at the observed radio frequency. Radio observations at lower frequencies could be used to measure the spectral index and to show the bend in the spectrum as a transition is made to optically thick conditions; such measurements would yield valuable diagnostic information on the physical conditions in the system.

Finally, we note that the ratio of inverse Compton losses to synchrotron losses is given by $u_{\gamma} / u_{B}$ where $u_{\gamma}$ is the energy density of photons and $u_{B}=B^{2} / 8 \pi$. Most of the energy density $u_{\gamma}$ in the V773 Tau A system arises from optical photons emitted by the two stars; to estimate this contribution, we divide the optical luminosity of the system, $3.93 L_{\odot}$ (Boden et al. 2007), by $4 \pi L^{2} c$ to obtain $u_{\gamma} \sim 0.010 \mathrm{erg} \mathrm{cm}^{-3}$. This value should be compared with the magnetic counterpart $u_{B} \sim 0.16 \mathrm{erg} \mathrm{cm}^{-3}$. Although inverse Compton losses are significantly smaller than synchrotron losses, they are not completely negligible. We suggest that it might be worthwhile to try to detect optical photons that have been scattered into the hard X-ray regime by the inverse Compton effect involving the synchrotron electrons that are deduced for the V773 Tau A system.

In summary, if our model is to have validity, two conditions must hold.

1. The synchrotron spectrum $S_{v}$ must not extend to frequencies much higher than the observed radio frequency of $v=90 \mathrm{GHz}$. At first sight, this requirement seems to be contradicted by the case of the DQ Tau binary, where an X-ray outburst coincided with a radio outburst (Getman et al. 2011; see also Salter et al. 2008). However, enhanced $\mathrm{X}$-ray emission also occurs in the case of solar radio outbursts, and the interpretation there is that the fast particles created by electromagnetic processes in an impulsive flare or coronal mass ejection travel down the field lines to the footpoints of a magnetic loop. The collisions that the fast electrons have with the solar atmosphere then produce optical and X-ray emission by brehmsstrahlung radiation. It would be interesting to know whether the X-ray outbursts in DQ Tau are synchrotron events (i.e., with a power-law spectrum and a high degree of linear polarization), or they are brehmsstrahlung events (i.e., with an optically thin thermal spectrum and no associated polarization), or they have the characteristics of high-energy photons that have been created by inverse Compton scattering.

2. Although the change of magnetospheric configuration in the V773 Tau A binary is occurring on the orbital timescale of weeks, the actual release of the stored energy occurs not on the orbital timescale (i.e., not as depicted in Figure 4), but on a much shorter burst timescale of hours (e.g., $t_{B} \sim 10^{4} \mathrm{~s}$ ). Again this property is consistent with the solar experience. Although the twisting of the coronal magnetic configuration originates in differential motions at the footpoints of the field in a solar atmosphere whose rotation rates at the pole and the equator are measured on a time of many weeks, the outbursts when they come occur on a timescale of minutes (for impulsive flares) or hours (for coronal mass ejections). The buildup of magnetic stresses may occur slowly and steadily (without instantaneous dissipation as assumed by the simplifying philosophy adopted at the beginning of our discussion (see Section 1)), but the relief of such stresses can appear suddenly and catastrophically as in solar flare events.

\subsection{Stellar Winds}

If the stars in the system have substantial stellar winds, their mechanical luminosity could compete with magnetically produced power from the interaction region. Scaling the 
mechanical luminosity $L_{\text {mech }}$ of the wind to the outflow rate from the Sun, we find

$$
\begin{aligned}
L_{\text {mech }}= & \frac{1}{2} \dot{M}_{w} v_{w}^{2} \approx 5 \times 10^{26} \mathrm{erg} \mathrm{s}^{-1}\left(\frac{\dot{M}_{w}}{10^{-14} M_{\odot} \mathrm{yr}^{-1}}\right) \\
& \times\left(\frac{v_{w}}{400 \mathrm{~km} \mathrm{~s}^{-1}}\right)^{2} .
\end{aligned}
$$

If the stars in V773 Tau A had solar-type winds, their mechanical luminosity would be more than 200 times smaller than the magnetic power scale of Equation (18). With a massloss rate $\dot{M} \approx 10^{-12} M_{\odot} \mathrm{yr}^{-1}$ for each star, wind power would be competitive with magnetic power, and such mass-loss rates are not beyond the capabilities of weak-line $\mathrm{T}$ Tauri stars.

In settings where stellar winds help shape magnetic field structures in young stars, the configuration could have both dipole and split-monopole components (Matt \& Pudritz 2005; Jardine et al. 1996), and this form has been proposed for the solar magnetic field (Banaszkiewicz et al. 1998). The field strength in a split-monopole declines as $B \propto r_{j}{ }^{-2}$, more slowly than a magnetic dipole, and collisionless shocks might play a stronger role in accelerating particles than magnetic reconnection. However, VLBI mapping for the V773 Tau A system (Massi et al. 2008) suggests otherwise; these authors interpret the magnetic field configurations as solar-like helmet streamers, where the outflow is not strong enough to overwhelm the fields. As a result, we suspect that the mass-loss rate in stellar winds in each of these weak-line T Tauri stars is substantially smaller than $\dot{M} \approx 10^{-12} M_{\odot} \mathrm{yr}^{-1}$. Indeed, observations suggest that typical mass-loss rates from weak-lined T Tauri stars are roughly $\dot{M} \sim 10^{-13} M_{\odot} \mathrm{yr}^{-1}$ (Guenther \& Emerson 1997).

Although the mechanical luminosity of the wind could compete with the magnetic power dissipated in the interaction region, the wind is not strong enough to greatly alter the magnetic field structure: the magnetic pressure in the interaction region is given by $P_{B}=B_{\text {int }}^{2} / 8 \pi$, where we expect $B_{\text {int }} \approx$ $2 \mathrm{G}$, so that $P_{B} \approx 0.16$ dyne $\mathrm{cm}^{-2}$. For comparison, the ram pressure of the wind, evaluated at the location of the interaction region, can be written in the form $P_{\text {ram }}=\dot{M} v_{w} /\left(4 \pi r^{2}\right)$. For a mass-loss rate $\dot{M}=10^{-12} M_{\odot} \mathrm{yr}^{-1}$, the ram pressure is only $P_{\text {ram }} \approx 5.6 \times 10^{-6}$ dyne $\mathrm{cm}^{-2}$, nearly 30,000 times smaller than the magnetic pressure. Closer to the star, the magnetic pressure dominates by an even larger margin. As a result, the magnetic field is strong enough that the wind cannot change the field configuration, and hence the use of dipole fields remains valid.

\subsection{Back Reaction}

In this scenario, magnetic energy is released when field lines connect as the stars move closer together. After periastron, as the stars separate, energy must be converted back into the magnetic fields in order to maintain the same dipole strength. This energy could come from either the stellar orbit or the stellar spins. Using Equations (11) and (16), we can estimate the total energy released per orbit

$$
\Delta E \approx\left(\frac{2 B_{*}^{2} R_{*}^{6}}{3 a_{0}^{3}}\right) \frac{\varepsilon\left(3+\varepsilon^{2}\right)}{\left(1-\varepsilon^{2}\right)^{3}},
$$

where $a_{\mathrm{o}}$ is the semimajor axis and we have assumed $\left|f_{\text {mean }}\right| \sim$ 1. Using parameters from Equation (13), we find the change in energy per orbit $\Delta E \approx 6 \times 10^{34} \mathrm{erg}$ (consistent with Equation (14)). The available mechanical energy in the orbit is the difference between the energies of the eccentric orbit and the circular orbit with the same angular momentum, about $3.5 \times 10^{45} \mathrm{erg}$. This energy supply can last $6 \times 10^{10}$ orbits, much longer than the age of the system. The back reaction on the stellar orbit is thus negligible.

Next we consider the stellar spins, which provide another possible source of energy for the magnetic interactions. Field lines connecting the two stars will be twisted by the combined motion of the orbit and the stellar spins to produce an azimuthal field. After one rotation period, these azimuthal field lines must reverse polarity along the line of centers joining the stars. This magnetic configuration naturally generates current sheets which in turn trigger reconnection events (because the field lines cannot be sheared indefinitely), consistent with the picture developed in this paper. Since the stars have periods of approximately $P_{\text {rot }} \sim 3$ days, both bodies have total spin kinetic energy $K_{\text {spin }} \sim I \omega^{2}$, where the moment of inertia $I=k M_{*} R_{*}^{2} \sim k\left(6 \times 10^{55}\right) \mathrm{g} \mathrm{cm}^{2}$, and where $k$ is a dimensionless constant. For example, for a polytrope with index $n=1.5$ (a good approximation for completely convective pre-mainsequence stars), one finds $k \approx 0.205$. The total energy stored in stellar rotation is then given by $K_{\text {spin }} \sim 7 \times 10^{45} \mathrm{erg}$, which is comparable to, but somewhat larger than, the available energy stored in the orbit. As a result, the spin energy supply can last for billions of orbits and the back reaction can (again) be neglected.

\section{CONCLUSION}

This paper shows that interactions between the magnetic fields of young eccentric binaries can provide significant power for accelerating fast electrons. The available interaction energy is large enough to produce the observed radio synchrotron signature in the well-studied system V773 Tau A. The energy stored in the magnetic fields of the system varies with orbital phase, with a maximum rate of energy dissipation occurring just before periastron (Section 2.3, Figure 5). This basic model can explain qualitatively the synchrotron radiation observed in the T Tauri binary V773 Tau A. However, the details as to how and why the radio flares occur with the energetics and timescales that they exhibit involve more elaborate considerations. These considerations include how magnetic field annihilation yields electric fields that accelerate electrons to relativistic energies and how these electrons are ultimately lost to the system, through synchrotron radiation, or brehmsstrahlung events, or escape from the system.

In the last regard, we began by assuming that all the field lines in the system close onto one star or the other. However, in Section 2.4 we presented the case that during peak outburst, the fast electrons in the V773 Tau A system may have enough pressure to open some of the field lines that join one star to the other. Through electrostatic forces, the flow of the electrons to the interstellar medium will carry a corresponding number of ions with them. The accelerated fast electrons that do not escape from the system will ultimately lose energy, either through collisions with ions (mostly at the polar caps of the two stars) or through synchrotron radiation. A surprising prediction of this model for the V773 Tau A system is that the synchrotron spectrum cuts off at radio frequencies only somewhat higher than the observed value of $90 \mathrm{GHz}$. Another prediction is that the X-ray emission in the young binary DQ Tau (with periastron $\sim 8 R_{*}$ ), which is correlated with the radio bursts (Getman et al. 2011), arises not from synchrotron emission, but probably has a brehmsstrahlung origin when fast particles associated with the electromagnetic acceleration stream to the footprints of the 
magnetic field and strike the surface of one of the stars in the system.

In closing, we note that the ideas presented in this paper can be tested. Perhaps the best way to study these phenomena further is through observational surveys of synchrotron radiation in a large sample of pre-main-sequence binary systems. In this model, the magnetic interaction energy $E_{\text {int }}$ and the idealized dissipated power $\mathcal{P}_{\text {mag }}$ are straightforward functions of the stellar field strengths, stellar radii, semimajor axis, and orbital eccentricity (Equations (11), (13), and (17)). The binary orbital elements and stellar properties can be measured independently of the synchrotron radiation. While hysteresis will make the synchrotron power more sporadic than the idealized dissipation described by Equation (17), the total energy release per orbit should be derivable from Equation (43).

We thank the Michigan Center for Theoretical Physics for helping to facilitate this collaboration, and thank Scott Gregory for useful discussions. We also thank Ron Taam for suggesting that we check whether inverse Compton scattering can play a role in V773 Tau A. This work was also supported by CONACyT J010/0654/10 (SL) and NASA Grant NNX11AK87G (FCA). Finally, we thank an anonymous referee for many useful comments that improved the manuscript.

\section{APPENDIX A}

\section{SOLID ANGLE OF THE MAGNETIC POLAR CAP}

As discussed in the text, and shown in Figure 4, the solid angle $A_{c}$ of the polar cap is given by the approximate empirical formula $A_{c} \approx 2 R_{*} / L$. This result can be understood as follows: half of the circumference of the polar cap arises from field lines that connect to a straight line of X-points defined by $(z=0, x=L)$, with $y$ running from $-\infty$ to $+\infty$. In spherical polar coordinates $(r, \theta, \varphi)$, the equation for a magnetic field line of a single dipole placed at the center of a star (the origin of the coordinate system) is defined by the equation

$$
r=r_{0} \sin ^{2} \theta
$$

where the field line crosses the equatorial plane at $r=r_{0}$. The line of X-points is given by the equation $r_{0} \cos \varphi=L$ with $\varphi$ running from $-\pi / 2$ to $+\pi / 2$. These lines map onto the surface of the star $r=R_{*}$ through the equation

$$
\sin ^{2} \theta=\left(\frac{R_{*}}{L}\right) \cos \varphi
$$

which describes a semi-curve, $\theta=\theta_{c}(\varphi)$, at the polar cap, where the argument varies from $\varphi=-\pi / 2$ to $\varphi=+\pi / 2$. In the expected regime $R_{*} / L \ll 1$, the contribution $A_{X}$ of the X-points to the solid angle of the polar cap is thus given by

$$
A_{X}=\int_{-\pi / 2}^{+\pi / 2} d \varphi \int_{0}^{\theta_{c}(\varphi)} \sin \theta d \theta=\int_{-\pi / 2}^{+\pi / 2}\left\{1-\cos \left[\theta_{c}(\varphi)\right]\right\} d \varphi .
$$

Equation (A2) implies

$$
\cos \left[\theta_{c}(\varphi)\right]=\left[1-\left(\frac{R_{*}}{L}\right) \cos \varphi\right]^{1 / 2} \approx 1-\left(\frac{R_{*}}{2 L}\right) \cos \varphi .
$$

Using this expression in Equation (A3) yields the approximation

$$
A_{X} \approx \frac{R_{*}}{L}
$$

From Figure 4, we find that the other semi-curve defined by the magnetic field loops that connect one star to the other via $z=+\infty$ contributes (approximately) an equal amount. The solid angle of the polar cap thus becomes

$$
A_{c} \approx \frac{2 R_{*}}{L}
$$

in agreement with the formula found empirically.

\section{APPENDIX B}

\section{GENERALIZED ELECTRON ENERGY DISTRIBUTION}

In this Appendix, we generalize the calculation of Section 2.4 to include a general power law form for the energy distribution of fast electrons, i.e.,

$$
n(\gamma) d \gamma=n_{0} \gamma^{-p} d \gamma
$$

where the index $p$ lies in the range $1 \leqslant p \leqslant 2.4$. The lower limit leads to a flat spectral energy distribution $v S_{v} \sim$ constant, whereas the upper limit is appropriate for collisionless shocks.

The number density $n_{T}$ of fast particles, integrated from $\gamma=1$ to $\gamma=\infty$, is finite, and is given by $n_{T}=n_{0} /(p-1)$ when $p>1$. However, the integrated electron pressure is given by

$$
P_{\mathrm{cr}}=\frac{1}{3} \int_{1}^{\infty} v \gamma m_{\mathrm{e}} v n(\gamma) d \gamma
$$

which diverges unless $p>2$. For $p<2$, one must introduce an upper cutoff in $\gamma$ to prevent the divergence of the pressure. For the case $p>2$, with $v \approx c$, the pressure $P_{\mathrm{cr}} \approx$ $n_{0} m_{\mathrm{e}} c^{2} /[3(p-2)]$.

The volume emissivity of synchrotron radiation takes the generalized form

$$
C_{1} r_{\mathrm{e}} n_{0} e B_{\perp}\left(\frac{v}{v_{\perp}}\right)^{-(p-1) / 2}
$$

where the dimensionless number $C_{1}$ is defined by

$$
C_{1}=\left(\frac{3^{p / 2} 2}{p+1}\right) \Gamma\left(\frac{p}{4}-\frac{1}{12}\right) \Gamma\left(\frac{p}{4}+\frac{19}{12}\right),
$$

where $\Gamma(\zeta)$ is the Gamma function. For optically thin synchrotron emission, the flux density at a distance $d$ from the source is given by

$$
S_{v}=\left[\frac{1}{3}\left(C_{1} r_{\mathrm{e}} n_{0} e B_{\perp}\right) L\right]\left(\frac{v}{v_{\perp}}\right)^{-(p-1) / 2}\left(\frac{L}{d}\right)^{2} .
$$

With $S_{v} \propto v^{-(p-1) / 2}$ in Equation (B5), the implied power $\mathcal{P}_{v}$, integrated from zero frequency to $v$, is given by

$$
\mathcal{P}_{\nu}=4 \pi d^{2} \int_{0}^{v} S_{v} d \nu=4 \pi d^{2}\left(\frac{2 \nu S_{v}}{3-p}\right) .
$$

For the system parameters of V773 Tau A, we find the power $\mathcal{P}_{v}=\left(1.53 \times 10^{30}\right) /(3-p)$ erg s $^{-1}$ for synchrotron emission 
Table 1

Electron Density and $90 \mathrm{GHz}$ Optical Depth

\begin{tabular}{lcccc}
\hline \hline$p$ & $C_{1}$ & $C_{2}$ & $\begin{array}{c}n_{T} \\
\left(\mathrm{~cm}^{-3}\right)\end{array}$ & $\tau_{v}$ \\
\hline 1.1 & 7.94 & 6.68 & $1.92 \times 10^{3}$ & $2.41 \times 10^{-4}$ \\
1.5 & 5.52 & 7.17 & $3.83 \times 10^{3}$ & $7.44 \times 10^{-5}$ \\
2.0 & 4.42 & 8.38 & $2.70 \times 10^{4}$ & $5.44 \times 10^{-5}$ \\
2.4 & 4.12 & 9.90 & $1.44 \times 10^{5}$ & $4.92 \times 10^{-5}$ \\
\hline
\end{tabular}

integrated from zero frequency to $v=90 \mathrm{GHz}$. Over a burst time $t_{B}=10^{4} \mathrm{~s}$, the released energy $E \approx\left(1.53 \times 10^{34}\right) /(3-p) \mathrm{erg}$ at radio frequencies up to $90 \mathrm{GHz}$.

Using $B_{\perp}=2 \mathrm{G}, v / v_{\perp}=(127)^{2}$, and $S_{v}=400 \mathrm{mJy}$ at $90 \mathrm{GHz}$, we find that the total number $n_{T}$ of fast electrons is given by

$$
n_{T}=\frac{n_{0}}{p-1}=\frac{939}{(p-1) C_{1}}(127)^{(p-1)} \mathrm{cm}^{-3} .
$$

Table 1 presents the number density $n_{T}$ and the parameter $C_{1}$ for a range of indices $p$. Note that for $2<p<2.4$, the density of fast electrons is consistent with the requirements arising from the reconnection-time argument (see the text).

The mean synchrotron optical depth through the source region at frequency $v$ is given by

$$
\tau_{\nu}=\frac{1}{3} C_{2} n_{0}\left(\frac{c r_{\mathrm{e}}}{v_{\perp}}\right) L\left(\frac{v}{v_{\perp}}\right)^{-(p+4) / 2},
$$

where

$$
C_{2}=3^{(p+1) / 2} \Gamma\left(\frac{3 p+2}{12}\right) \Gamma\left(\frac{3 p+22}{12}\right) .
$$

Table 1 also presents the optical depth $\tau_{v}$ (at $v=90 \mathrm{GHz}$ ) and the parameter $C_{2}$ for varying indices $p$. For all plausible energy distributions of the fast electrons, those with $p>1$, the synchrotron radiation is optically thin at the observed radio frequency $v=90 \mathrm{GHz}$. Note that radio observations at lower frequencies will provide important additional information regarding the physical conditions in the system (see the text).

\section{REFERENCES}

Banaszkiewicz, M., Axford, W. I., \& McKenzie, J. F. 1998, A\&A, 337, 940

Boden, A. F., Torres, G., Sargent, A. I., et al. 2007, ApJ, 670, 1214

Dreicer, H. 1959, Phys. Rev., 115, 238

Getman, K. V., Broos, P. S., Salter, D. M., Garmire, G. P., \& Hogerheijde, M. R. 2011, ApJ, 730, 6

Goldreich, P., \& Lynden-Bell, D. 1969, ApJ, 156, 59

Gregory, S. G., Jardine, M., Gray, C. G., \& Donati, J.-F. 2010, Rep. Prog. Phys., 73, 126901

Guenther, E. W., \& Emerson, J. P. 1997, A\&A, 321, 803

Jackson, J. D. 1962, Classical Electrodynamics (New York: Wiley)

Jardine, M., Allen, H. R., \& Pollock, A. M. T. 1996, A\&A, 314, 594

Johns-Krull, C. M. 2009, in IAU Symp. 259, Cosmic Magnetic Fields: From Planets, to Stars and Galaxies, ed. K. G. Strassmeier, A. G. Kosovichev, \& J. E. Beckman (Cambridge: Cambridge Univ. Press), 345

Königl, A. 1991, ApJ, 370, L39

Massi, M., Forbrich, J., Menten, K. M., et al. 2006, A\&A, 453, 959

Massi, M., Ros, E., Menten, K. M., et al. 2008, A\&A, 480, 489

Matt, S., \& Pudritz, R. E. 2005, ApJ, 632, 135

Melrose, D. B. 1972, Ap\&SS, 18, 267

Murray, C. D., \& Dermott, S. F. 1999, Solar System Dynamics (Cambridge: Cambridge Univ. Press)

Phillips, R. B., Lonsdale, C. J., Feigelson, E. D., \& Deeny, B. D. 1996, AJ, 111, 918

Razin, V. A. 1960, Iz. Vys. Ucheb. Zaved. Radiofiz., 3, 584

Salter, D. M., Hogerheijde, M. R., \& Blake, G. A. 2008, A\&A, 492, L21

Shu, F. H. 1991, Radiation: The Physics of Astrophysics, Vol. I (Mill Valley, CA: Univ. Science Books)

Shu, F., Najita, J., Ostriker, E., et al. 1994, ApJ, 429, 781

Simon, M. 1969, ApJ, 158, 865

Spitzer, L. 1978, Physics Processes in the Interstellar Medium (New York: Wiley)

Tandberg-Hanssen, E., \& Emslie, A. G. 2009, The Physics of Solar Flares (Cambridge: Cambridge Univ. Press)

Torres, R. M., Loinard, L., Mioduszewski, A. J., et al. 2011, ApJ, submitted

Tsytovich, V. N. 1951, Vestn. Mosk. Univ., 11, 27

Welty, A. D. 1995, AJ, 110, 776 